\title{
Quantum coherence and CPT symmetry tests in the neutral kaon system at KLOE
}

\author{
Antonio Di Domenico* \\ Dipartimento di Fisica, "Sapienza” Università di Roma, \\ and I.N.F.N. Sezione di Roma, P.le A. Moro, 2, I-00185 Rome, Italy E-mail: \\ antonio.didomenico@roma1. infn.it
}

\section{on behalf of the KLOE collaboration ${ }^{\dagger}$}

\begin{abstract}
The neutral kaon system offers a unique possibility to perform fundamental tests of $C P T$ invariance, as well as of the basic principles of quantum mechanics. The most recent limits obtained by the KLOE experiment at the DA $\Phi$ NE $e^{+} e^{-}$collider on several kinds of possible CPT violation and decoherence mechanisms, which in some cases might be justified in a quantum gravity framework, are reviewed. No deviation from the expectations of quantum mechanics and $C P T$ symmetry is observed, while the precision of the measurements, in some cases, reaches the interesting Planck scale region. Finally, prospects for this kind of experimental studies at KLOE-2 are presented.
\end{abstract}

2009 KAON International Conference

June 09 - 12, 2009

Tsukuba, Japan

${ }^{*}$ Speaker.

${ }^{\dagger}$ The KLOE collaboration: F. Ambrosino, A. Antonelli, M. Antonelli, F. Archilli, P. Beltrame, G. Bencivenni, C. Bini, C. Bloise, S. Bocchetta, F. Bossi, P. Branchini, G. Capon, D. Capriotti, T. Capussela, F. Ceradini, P. Ciambrone, E. De Lucia, A. De Santis, P. De Simone, G. De Zorzi, A. Denig, A. Di Domenico, C. Di Donato, B. Di Micco, M. Dreucci, G. Felici, S. Fiore, P. Franzini, C. Gatti, P. Gauzzi, S. Giovannella, E. Graziani, M. Jacewicz, V. Kulikov, G. Lanfranchi, J. Lee-Franzini, M. Martini, P. Massarotti, S. Meola, S. Miscetti, M. Moulson, S. Müller, F. Murtas, M. Napolitano, F. Nguyen, M. Palutan, A. Passeri, V. Patera, P. Santangelo, B. Sciascia, A. Sibidanov, T. Spadaro, L. Tortora, P. Valente, G. Venanzoni, R. Versaci. 


\section{Introduction}

The three discrete symmetries of quantum mechanics, $C$ (charge conjugation), $P$ (parity), and $T$ (time reversal) are known to be violated in nature, both singly and in pairs. Only the combination of the three - CPT (in any order) - appears to be an exact symmetry of nature. This fact has a very solid theoretical motivation in the $C P T$ theorem, a rigorous proof of which can be found in Refs. $[1,2,3,4]$ (see also Refs. [5, 6, 7] for some recent developments). This theorem ensures that exact $C P T$ invariance holds for any quantum field theory formulated on flat space-time assuming (1) Lorentz invariance, (2) Locality, and (3) Unitarity (i.e. conservation of probability). Testing the validity of $C P T$ invariance therefore probes the most fundamental assumptions of our present understanding of particles and their interactions.

The neutral kaon doublet is one of the most intriguing systems in nature. During its time evolution a neutral kaon oscillates between its particle and antiparticle states with a beat frequency $\Delta m \approx 5.3 \times 10^{9} \mathrm{~s}^{-1}$, where $\Delta m$ is the small mass difference between the exponentially decaying states $\mathrm{K}_{\mathrm{L}}$ and $\mathrm{K}_{\mathrm{S}}$. The fortunate coincidence that $\Delta m$ is about half the decay width of $\mathrm{K}_{\mathrm{S}}$ makes it possible to observe a variety of intricate interference phenomena in the time evolution and decay of neutral kaons. In turn, such observations enable us to test quantum mechanics, the interplay of different conservation laws and the validity of various symmetry principles. In particular the extreme sensitivity of the neutral kaon system to a variety of $C P T$-violating effects makes it one of the best candidates for an accurate experimental test of this symmetry. As a figure of merit, the fractional mass difference $\left(m_{\mathrm{K}^{0}}-m_{\overline{\mathrm{K}}^{0}}\right) / m_{\mathrm{K}^{0}}$ can be considered: it can be measured at the level of $\mathscr{O}\left(10^{-18}\right)$ for neutral kaons, while, for comparison, a limit of $\mathscr{O}\left(10^{-14}\right)$ can be reached on the corresponding quantity for the $\mathrm{B}^{0}-\overline{\mathrm{B}}^{0}$ system, and only of $\mathscr{O}\left(10^{-8}\right)$ for proton-antiproton [8]. Interferometric methods applied to neutral kaon pairs at a $\phi$-factory add new possibilities for this kind of tests [9].

\section{CPT test from unitarity}

The real part of the complex parameter $\delta$, describing $C P T$ violation in $\mathrm{K}^{0}-\overline{\mathrm{K}}^{0}$ mixing, has been measured by the CPLEAR collaboration studying the time behaviour of semileptonic decays from initially tagged $\mathrm{K}^{0}$ and $\overline{\mathrm{K}}^{0}$ mesons [10]:

$$
\Re \delta=\left(0.30 \pm 0.33_{\text {stat }} \pm 0.06_{\text {syst }}\right) \times 10^{-3} .
$$

One of the most precise and significant tests of the CPT symmetry comes from the unitarity relation, originally derived by Bell and Steinberger [11]:

$$
\begin{aligned}
& \left(\frac{\Gamma_{S}+\Gamma_{L}}{\Gamma_{S}-\Gamma_{L}}+i \tan \phi_{S W}\right)\left[\frac{\Re \varepsilon}{1+|\varepsilon|^{2}}-i \mathfrak{I} \delta\right] \\
& =\frac{1}{\Gamma_{S}-\Gamma_{L}} \sum_{f} A^{*}\left(K_{S} \rightarrow f\right) A\left(K_{L} \rightarrow f\right) \equiv \sum_{f} \alpha_{f},
\end{aligned}
$$

where $\varepsilon$ is the usual complex parameter describing $C P$ violation in $\mathrm{K}^{0}-\overline{\mathrm{K}}^{0}$ mixing; $\Gamma_{S}$ and $\Gamma_{L}$ are the widths of the physical states $\mathrm{K}_{\mathrm{S}}$ and $\mathrm{K}_{\mathrm{L}} ; \phi_{S W}$ is the superweak phase; $A\left(K_{i} \rightarrow f\right)$ is the decay amplitude of the state $K_{i}$ into final state $f$, and the sum runs over all possible final states. 
The above relationship can be used to bound the parameter $\mathfrak{I} \delta$, after having provided all the $\alpha_{i}$ parameters, $\Gamma_{S}, \Gamma_{L}$, and $\phi_{S W}$ as inputs. Using several measurements from the KLOE experiment [12], values from the Particle Data Group (PDG), and a combined fit of KLOE and CPLEAR data, the following result is obtained [8]:

$$
\mathfrak{I} \delta=(-0.6 \pm 1.9) \times 10^{-5},
$$

which is the most stringent limit on $\mathfrak{I} \delta^{1}$, the main limiting factor of this result being the uncertainty on the phase $\phi_{+-}$entering in the parameter $\alpha_{\pi^{+} \pi^{-}}$.

The limits on $\mathfrak{I} \delta$ and $\mathfrak{R} \delta$ can be used to constrain the mass and width difference between $\mathrm{K}^{0}$ and $\overline{\mathrm{K}}^{0}$. In the limit $\Gamma_{\mathrm{K}^{0}}-\Gamma_{\overline{\mathrm{K}}^{0}}=0$, i.e. neglecting $C P T$-violating effects in the decay amplitudes, the best bound on the neutral kaon mass difference is obtained:

$$
\left|m_{\mathrm{K}^{0}}-m_{\overline{\mathrm{K}}^{0}}\right|<5.1 \times 10^{-19} \mathrm{GeV} \quad \text { at } 95 \% \mathrm{CL} .
$$

A preliminary update including the latest results on $\phi_{+-}$by the $\mathrm{KTeV}$ collaboration [13] yields slightly improved results [14]:

$$
\begin{gathered}
\mathfrak{I} \delta=(-0.1 \pm 1.4) \times 10^{-5} \\
\left|m_{\mathrm{K}^{0}}-m_{\overline{\mathrm{K}}^{0}}\right|<4.0 \times 10^{-19} \mathrm{GeV} \quad \text { at } 95 \% \mathrm{CL} .
\end{gathered}
$$

\section{CPT and QM tests}

DAФNE, the Frascati $\phi$-factory, is an $e^{+} e^{-}$collider working at a center of mass energy of $\sqrt{s} \sim 1020 \mathrm{MeV}$, corresponding to the peak of the $\phi$ resonance. The $\phi$ production cross section is $\sim 3 \mu \mathrm{b}$, and its decay into $\mathrm{K}^{0} \overline{\mathrm{K}}^{0}$ pairs has a branching fraction of $34 \%$. The neutral kaon pair is produced in a coherent quantum state with quantum numbers $J^{P C}=1^{--}$:

$$
|i\rangle=\frac{1}{\sqrt{2}}\left\{\left|\mathrm{~K}^{0}\right\rangle\left|\overline{\mathrm{K}}^{0}\right\rangle-\left|\overline{\mathrm{K}}^{0}\right\rangle\left|\mathrm{K}^{0}\right\rangle\right\}=\frac{N}{\sqrt{2}}\left\{\left|\mathrm{~K}_{\mathrm{S}}\right\rangle\left|\mathrm{K}_{\mathrm{L}}\right\rangle-\left|\mathrm{K}_{\mathrm{L}}\right\rangle\left|\mathrm{K}_{\mathrm{S}}\right\rangle\right\}
$$

where $N=\sqrt{\left(1+\left|\varepsilon_{S}\right|^{2}\right)\left(1+\left|\varepsilon_{L}\right|^{2}\right)} /\left(1-\varepsilon_{S} \varepsilon_{L}\right) \simeq 1$ is a normalization factor, and $\varepsilon_{S, L}=\varepsilon \pm \delta$.

The detection of a kaon at large (small) times tags a $\mathrm{K}_{\mathrm{S}}\left(\mathrm{K}_{\mathrm{L}}\right)$ in the opposite direction.

The KLOE detector consists mainly of a large volume drift chamber[15] surrounded by an electromagnetic calorimeter[16], both inside a superconducting coil providing an axial $0.52 \mathrm{~T}$ magnetic field.

At $\mathrm{KLOE}$ a $\mathrm{K}_{\mathrm{S}}$ is tagged by identifying the interaction of the $\mathrm{K}_{\mathrm{L}}$ in the calorimeter $\left(\mathrm{K}_{\mathrm{L}}\right.$-crash), while a $\mathrm{K}_{\mathrm{L}}$ is tagged by detecting a $\mathrm{K}_{\mathrm{S}} \rightarrow \pi^{+} \pi^{-}$decay near the interaction point (IP).

KLOE completed the data taking in March 2006 with a total integrated luminosity $\mathrm{L} \sim 2.5 \mathrm{fb}^{-1}$, corresponding to $\sim 7.5 \times 10^{9} \phi$-mesons produced.

The quantum interference between the two kaons initially in the entangled state in eq.(3.1) and decaying in the $C P$ violating channel $\phi \rightarrow \mathrm{K}_{\mathrm{S}} \mathrm{K}_{\mathrm{L}} \rightarrow \pi^{+} \pi^{-} \pi^{+} \pi^{-}$, has been observed for the first time by the KLOE collaboration [17], analyzing a data sample corresponding to $L \simeq 380 \mathrm{pb}^{-1}$.

\footnotetext{
${ }^{1}$ The result $\Re \varepsilon=(161.2 \pm 0.6) \times 10^{-5}$, which is obtained in the same analysis, is not relevant for the discussion here.
} 
Here the final results obtained in the analysis of a different and larger data sample, corresponding to $L \simeq 1.5 \mathrm{fb}^{-1}$, are presented [18]. The measured $\Delta t$ distribution, with $\Delta t$ the absolute value of the time difference of the two $\pi^{+} \pi^{-}$decays, can be fitted with the distribution:

$$
\begin{gathered}
I\left(\pi^{+} \pi^{-}, \pi^{+} \pi^{-} ; \Delta t\right) \propto e^{-\Gamma_{L} \Delta t}+e^{-\Gamma_{S} \Delta t} \\
-2\left(1-\zeta_{S L}\right) e^{-\frac{\left(\Gamma_{S}+\Gamma_{L}\right)}{2} \Delta t} \cos (\Delta m \Delta t),
\end{gathered}
$$

where the quantum mechanical expression in the $\left\{\mathrm{K}_{\mathrm{S}}, \mathrm{K}_{\mathrm{L}}\right\}$ basis has been modified with the introduction of a decoherence parameter $\zeta_{S L}$, and a factor $\left(1-\zeta_{S L}\right)$ multiplying the interference term. Analogously, a $\zeta_{0 \overline{0}}$ parameter can be defined in the $\left\{\mathrm{K}^{0}, \overline{\mathrm{K}}^{0}\right\}$ basis [19]. After having included resolution and detection efficiency effects, having taken into account the background due to coherent and incoherent $\mathrm{K}_{\mathrm{S}}$-regeneration on the beam pipe wall, the small contamination of non-resonant $e^{+} e^{-} \rightarrow \pi^{+} \pi^{-} \pi^{+} \pi^{-}$events, and keeping $\Delta m, \Gamma_{S}$ and $\Gamma_{L}$ fixed to the PDG values, the fit is performed on the $\Delta t$ distribution, as shown in Fig.1.

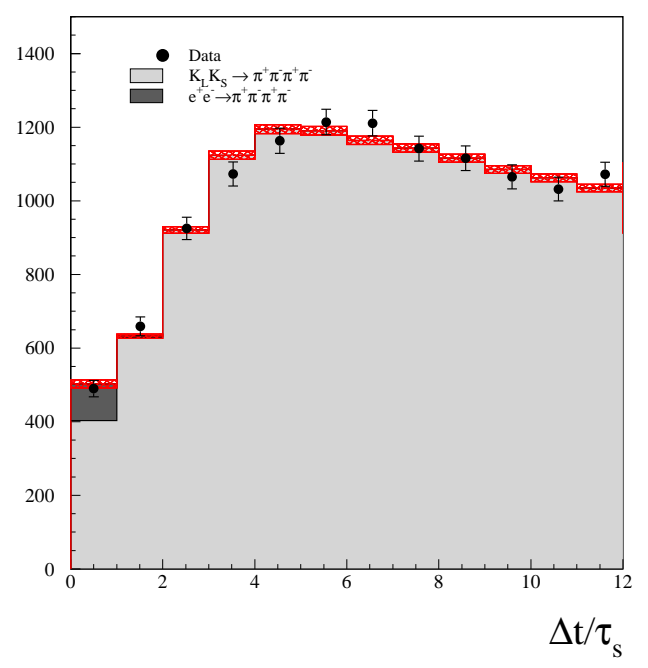

Figure 1: Fit of the measured $I\left(\pi^{+} \pi^{-}, \pi^{+} \pi^{-} ; \Delta t\right)$ distribution. The black points with errors are data and the solid histogram is the fit result. The uncertainty arising from the efficiency correction is shown as the hatched area.

The results are [18]:

$$
\begin{aligned}
& \zeta_{S L}=\left(0.3 \pm 1.8_{\text {stat }} \pm 0.6_{\text {syst }}\right) \times 10^{-2} \\
& \zeta_{0 \overline{0}}=\left(1.4 \pm 9.5_{\text {stat }} \pm 3.8_{\text {syst }}\right) \times 10^{-7}
\end{aligned}
$$

compatible with the prediction of quantum mechanics, i.e. $\zeta_{S L}=\zeta_{0 \overline{0}}=0$, and no decoherence effect. In particular the result on $\zeta_{0 \overline{0}}$ has a high precision, $\mathscr{O}\left(10^{-6}\right)$, due to the $C P$ suppression present in the specific decay channel; it is an improvement by five orders of magnitude over the previous limit, obtained by Bertlmann and co-workers [19] in a re-analysis of CPLEAR data [20]. This result can also be compared to a similar one recently obtained in the B meson system [21], where an accuracy of $\mathscr{O}\left(10^{-2}\right)$ has been reached.

At a microscopic level, in a quantum gravity picture, space-time might be subjected to inherent non-trivial quantum metric and topology fluctuations at the Planck scale $\left(\sim 10^{-33} \mathrm{~cm}\right)$, called 
generically space-time foam, with associated microscopic event horizons. This space-time structure would lead to pure states evolving to mixed states, i.e. the decoherence of apparently isolated matter systems [22]. This decoherence, in turn, necessarily implies, by means of a theorem [23], $C P T$ violation, in the sense that the quantum mechanical operator generating $C P T$ transformations cannot be consistently defined.

A model for decoherence can be formulated $[24,25]$ in which a single kaon is described by a density matrix $\rho$ that obeys a modified Liouville-von Neumann equation:

$$
\frac{d \rho}{d t}=-i \mathbf{H} \rho+i \rho \mathbf{H}^{\dagger}+L(\rho ; \alpha, \beta, \gamma)
$$

where $\mathbf{H}$ is the neutral kaon effective Hamiltonian, and the extra term $L(\rho ; \alpha, \beta, \gamma)$ would induce decoherence in the system, and depends on three real parameters, $\alpha, \beta$ and $\gamma$, which violate $C P T$ symmetry and quantum mechanics (they satisfy the inequalities $\alpha, \gamma>0$ and $\alpha \gamma>\beta^{2}$ - see Refs. [24, 25]). They have units of mass and are presumed to be at most $\mathscr{O}\left(m_{K}^{2} / M_{\text {Planck }}\right) \sim$ $2 \times 10^{-20} \mathrm{GeV}$, where $M_{\text {Planck }}=1 \sqrt{G_{N}}=1.22 \times 10^{19} \mathrm{GeV}$ is the Planck mass.

The CPLEAR collaboration, studying the time behaviour of single neutral kaon decays to $\pi^{+} \pi^{-}$and $\pi e v$ final states, obtained the following results [26]:

$$
\begin{aligned}
\alpha & =(-0.5 \pm 2.8) \times 10^{-17} \mathrm{GeV} \\
\beta & =(2.5 \pm 2.3) \times 10^{-19} \mathrm{GeV} \\
\gamma & =(1.1 \pm 2.5) \times 10^{-21} \mathrm{GeV}
\end{aligned}
$$

The KLOE collaboration, studying the same $I\left(\pi^{+} \pi^{-}, \pi^{+} \pi^{-} ; \Delta t\right)$ distribution as in the $\zeta$ parameters analysis, in the simplifying hypothesis of complete positivity ${ }^{2}$ [27], i.e. $\alpha=\gamma$ and $\beta=0$, obtained the following result:

$$
\gamma=\left(0.7 \pm 1.2_{\text {stat }} \pm 0.3_{\text {syst }}\right) \times 10^{-21} \mathrm{GeV},
$$

All results are compatible with no $C P T$ violation, while the sensitivity approaches the interesting level of $\mathscr{O}\left(10^{-20} \mathrm{GeV}\right)$.

As discussed above, in a quantum gravity framework inducing decoherence, the $C P T$ operator is ill-defined. This consideration might have intriguing consequences in correlated neutral kaon states, where the resulting loss of particle-antiparticle identity could induce a breakdown of the correlation in state (3.1) imposed by Bose statistics [28, 29]. As a result the initial state (3.1) can be parametrized in general as:

$$
|i\rangle=\frac{1}{\sqrt{2}}\left[\left|\mathrm{~K}^{0}\right\rangle\left|\overline{\mathrm{K}}^{0}\right\rangle-\left|\overline{\mathrm{K}}^{0}\right\rangle\left|\mathrm{K}^{0}\right\rangle+\omega\left(\left|\mathrm{K}^{0}\right\rangle\left|\overline{\mathrm{K}}^{0}\right\rangle+\left|\overline{\mathrm{K}}^{0}\right\rangle\left|\mathrm{K}^{0}\right\rangle\right)\right]
$$

where $\omega$ is a complex parameter describing a completely novel $C P T$ violation phenomenon, not included in previous analyses. Its order of magnitude could be at most

$$
|\omega| \sim\left[\left(m_{K}^{2} / M_{\text {Planck }}\right) / \Delta \Gamma\right]^{1 / 2} \sim 10^{-3}
$$

\footnotetext{
${ }^{2}$ This hypothesis, reducing the number of free parameters, makes the fit of the experimental distribution easier, even though it is not strictly necessary from the analysis point of view.
} 
with $\Delta \Gamma=\Gamma_{S}-\Gamma_{L}$.

A similar analysis performed by the KLOE collaboration on the same $I\left(\pi^{+} \pi^{-}, \pi^{+} \pi^{-} ; \Delta t\right)$ distribution as before, including in the fit the modified initial state eq.(3.7), yields the first measurement of the complex parameter $\omega$ :

$$
\begin{aligned}
& \mathfrak{R}(\omega)=\left(-1.6_{-2.1}^{+3.0} \text { stat }^{+} \pm 0.4_{\text {syst }}\right) \times 10^{-4} \\
& \mathfrak{I}(\omega)=\left(-1.7_{-3.0_{\text {stat }}}^{+3.3} \pm 1.2_{\text {syst }}\right) \times 10^{-4},
\end{aligned}
$$

with $|\omega|<1.0 \times 10^{-3}$ at $95 \%$ C.L. and an accuracy that already reaches the interesting Planck scale region.

\section{CPT violation and Lorentz symmetry breaking}

$C P T$ invariance holds for any realistic Lorentz-invariant quantum field theory. However a very general theoretical possibility for $C P T$ violation is based on spontaneous breaking of Lorentz symmetry [30,31,32], which appears to be compatible with the basic tenets of quantum field theory and retains the property of gauge invariance and renormalizability (Standard Model Extensions SME). In SME for neutral kaons, $C P T$ violation manifests to lowest order only in the parameter $\delta$, and exhibits a dependence on the 4-momentum of the kaon:

$$
\delta \approx i \sin \phi_{S W} e^{i \phi_{S W}} \gamma_{K}\left(\Delta a_{0}-\overrightarrow{\beta_{K}} \cdot \Delta \vec{a}\right) / \Delta m
$$

where $\gamma_{K}$ and $\vec{\beta}_{K}$ are the kaon boost factor and velocity in the observer frame, and $\Delta a_{\mu}$ are four $C P T$ - and Lorentz-violating coefficients for the two valence quarks in the kaon.

Following Ref. [31], the time dependence arising from the rotation of the Earth can be explicitly displayed in eq. (4.1) by choosing a three-dimensional basis $(\hat{X}, \hat{Y}, \hat{Z})$ in a non-rotating frame, with the $\hat{Z}$ axis along the Earth's rotation axis, and a basis $(\hat{x}, \hat{y}, \hat{z})$ for the rotating (laboratory) frame. The $C P T$ violating parameter $\delta$ may then be expressed as:

$$
\begin{aligned}
\delta= & \frac{1}{2 \pi} \int_{0}^{2 \pi} \delta\left(\vec{p}, t_{\text {sid }}\right) d \phi=\frac{i \sin \phi_{S W} e^{i \phi_{S W}}}{\Delta m} \gamma_{K}\left\{\Delta a_{0}+\beta_{K} \Delta a_{Z} \cos \theta \cos \chi\right. \\
& \left.+\beta_{K}\left(\Delta a_{Y} \sin \chi \cos \theta \sin \Omega t_{s i d}+\Delta a_{X} \sin \chi \cos \theta \cos \Omega t_{s i d}\right)\right\}
\end{aligned}
$$

where $t_{\text {sid }}$ is the sidereal time, $\Omega$ is the Earth's sidereal frequency, $\cos \chi=\hat{z} \cdot \hat{z}, \theta$ and $\phi$ are the conventional polar and azimuthal angles defined in the laboratory frame about the $\hat{z}$ axis, and an integration on the azimuthal angle $\phi$ has been performed, assuming a symmetric decay distribution in this variable ${ }^{3}$. The sensitivity to the four $\Delta a_{\mu}$ parameters can be very different for fixed target and collider experiments, showing complementary features [31].

At KLOE the $\Delta a_{0}$ parameter can be evaluated through the difference of the semileptonic charge asymmetries:

$$
A_{S, L}=\frac{\Gamma\left(\mathrm{K}_{\mathrm{S}, \mathrm{L}} \rightarrow \pi^{-} l^{+} v\right)-\Gamma\left(\mathrm{K}_{\mathrm{S}, \mathrm{L}} \rightarrow \pi^{+} l^{-} \bar{v}\right)}{\Gamma\left(\mathrm{K}_{\mathrm{S}, \mathrm{L}} \rightarrow \pi^{-} l^{+} v\right)+\Gamma\left(\mathrm{K}_{\mathrm{S}, \mathrm{L}} \rightarrow \pi^{+} l^{-} \bar{v}\right)}
$$

\footnotetext{
${ }^{3}$ Although not necessary, this assumption is taken here in order to simplify formulas.
} 
by performing the measurement of each asymmetry with a symmetric integration over the polar angle $\theta$, thus averaging to zero any possible contribution from the terms proportional to $\cos \theta$ in eq.(4.2):

$$
A_{S}-A_{L} \simeq\left[\frac{4 \Re\left(i \sin \phi_{S W} e^{i \phi_{S W}}\right) \gamma_{K}}{\Delta m}\right] \Delta a_{0} .
$$

In this way a first preliminary evaluation of the $\Delta a_{0}$ parameter can be obtained by KLOE $[9,33]$ :

$$
\Delta a_{0}=(0.4 \pm 1.8) \times 10^{-17} \mathrm{GeV} .
$$

With the analysis of the full KLOE data sample $\left(L=2.5 \mathrm{fb}^{-1}\right)$ an accuracy $\sigma\left(\Delta a_{0}\right) \sim 7 \times 10^{-18} \mathrm{GeV}$ could be reached.

At KLOE the $\Delta a_{X, Y, Z}$ parameters can be evaluated performing a sidereal time dependent analysis of the asymmetry:

$$
A(\Delta t)=\frac{N^{+}-N^{-}}{N^{+}+N^{-}}
$$

with $N^{+}=I\left(\pi^{+} \pi^{-}(+), \pi^{+} \pi^{-}(-) ; \Delta t>0\right)$ and $N^{-}=I\left(\pi^{+} \pi^{-}(+), \pi^{+} \pi^{-}(-) ; \Delta t<0\right)$, where the two identical final states are distinguished by their emission in the forward $(\cos \theta>0)$ or backward $(\cos \theta<0)$ hemispheres (denoted by the symbols + and - , respectively), and $\Delta t$ is the time difference between $(+)$ and $(-) \pi^{+} \pi^{-}$decays. A preliminary analysis based on a data sample corresponding to an integrated luminosity $\mathrm{L} \sim 1 \mathrm{fb}^{-1}$ yields the following results $[9,33,34]$ :

$$
\begin{gathered}
\Delta a_{X}=(-6.3 \pm 6.0) \times 10^{-18} \mathrm{GeV} \\
\Delta a_{Y}=(2.8 \pm 5.9) \times 10^{-18} \mathrm{GeV} \\
\Delta a_{Z}=(2.4 \pm 9.7) \times 10^{-18} \mathrm{GeV} .
\end{gathered}
$$

A preliminary measurement performed by the $\mathrm{KTeV}$ collaboration [35] based on the search for sidereal time variation of the phase $\phi_{+-}$constrains $\Delta a_{X}$ and $\Delta a_{Y}$ to less than $9.2 \times 10^{-22} \mathrm{GeV}$ at $90 \%$ C.L. These results can also be compared to similar ones recently obtained in the B meson system [36], where an accuracy on the $\Delta a_{\mu}^{B}$ parameters of $\mathscr{O}\left(10^{-13} \mathrm{GeV}\right)$ has been reached.

\section{Future plans}

A proposal $[37,38,39]$ has been presented for a physics program to be carried out with an upgraded KLOE detector, KLOE-2, at an upgraded DAФNE machine, which has been assumed to deliver an integrated luminosity up to $20 \div 50 \mathrm{fb}^{-1}$. The major upgrade of the KLOE detector would consist in the addition of an inner tracker for the improvement of decay vertex resolution, therefore improving the resolution on $\Delta t$, and consequently the sensitivity on several parameters based on kaon interferometry measurements.

The KLOE-2 program concerning neutral kaon interferometry is summarized in table 1 , where the KLOE-2 statistical sensitivities on the main parameters which can be extracted from kaon decay time distributions $I\left(f_{1}, f_{2} ; \Delta t\right)$ (with different choices of final states $f_{1}$ and $\left.f_{2}\right)$ are listed in the hypothesis of an integrated luminosity $L=50 \mathrm{fb}^{-1}$, and compared to the best present measurements. Improvements of about one order of magnitude in almost all present limits on $C P T$ violation and decoherence parameters are expected. 
Table 1: KLOE-2 statistical sensitivities on several parameters.

\begin{tabular}{|c|c|c|c|c|}
\hline$f_{1}$ & $f_{2}$ & Parameter & Present best measurement & KLOE-2 $\left(50 \mathrm{fb}^{-1}\right)$ \\
\hline$K_{S} \rightarrow \pi e v$ & & $A_{S}$ & $(1.5 \pm 11) \times 10^{-3}$ & $\pm 1 \times 10^{-3}$ \\
\hline$\pi^{+} \pi^{-}$ & $\pi l v$ & $A_{L}$ & $(3322 \pm 58 \pm 47) \times 10^{-6}$ & $\pm 25 \times 10^{-6}$ \\
\hline$\pi^{+} \pi^{-}$ & $\pi^{0} \pi^{0}$ & $\Re \frac{\varepsilon^{\prime}}{\varepsilon}$ & $(1.65 \pm 0.26) \times 10^{-3}($ PDG fit $)$ & $\pm 0.2 \times 10^{-3}$ \\
\hline$\pi^{+} \pi^{-}$ & $\pi^{0} \pi^{0}$ & $\mathfrak{I} \frac{\varepsilon^{\prime}}{\varepsilon}$ & $(-1.2 \pm 2.3) \times 10^{-3}$ (PDG fit) & $\pm 3 \times 10^{-3}$ \\
\hline$\pi^{+} l^{-} \bar{v}$ & $\pi^{-} l^{+} v$ & $\left(\Re \delta+\Re x_{-}\right)$ & $\begin{array}{l}\Re \delta=(0.25 \pm 0.23) \times 10^{-3}(\mathrm{PDG}) \\
\mathfrak{R} x_{-}=(-4.2 \pm 1.7) \times 10^{-3}(\mathrm{PDG})\end{array}$ & $\pm 0.2 \times 10^{-3}$ \\
\hline$\pi^{+} l^{-} \bar{v}$ & $\pi^{-} l^{+} v$ & $\left(\mathfrak{I} \delta+\mathfrak{I} x_{+}\right)$ & $\begin{array}{l}\mathfrak{I} \delta=(-0.6 \pm 1.9) \times 10^{-5}(\mathrm{PDG}) \\
\mathfrak{I} x_{+}=(0.2 \pm 2.2) \times 10^{-3}(\mathrm{PDG})\end{array}$ & $\pm 3 \times 10^{-3}$ \\
\hline$\pi^{+} \pi^{-}$ & $\pi^{+} \pi^{-}$ & $\Delta m$ & $5.288 \pm 0.043 \times 10^{9} s^{-1}$ & $\pm 0.03 \times 10^{9} s^{-1}$ \\
\hline$\pi^{+} \pi^{-}$ & $\pi^{+} \pi^{-}$ & $\zeta_{S L}$ & $(0.3 \pm 1.9) \times 10^{-2}$ & $\pm 0.2 \times 10^{-2}$ \\
\hline$\pi^{+} \pi^{-}$ & $\pi^{+} \pi^{-}$ & $\zeta_{0 \overline{0}}$ & $(0.1 \pm 1.0) \times 10^{-6}$ & $\pm 0.1 \times 10^{-6}$ \\
\hline$\pi^{+} \pi^{-}$ & $\pi^{+} \pi^{-}$ & $\alpha$ & $(-0.5 \pm 2.8) \times 10^{-17} \mathrm{GeV}$ & $\pm 2 \times 10^{-17} \mathrm{GeV}$ \\
\hline$\pi^{+} \pi^{-}$ & $\pi^{+} \pi^{-}$ & $\beta$ & $(2.5 \pm 2.3) \times 10^{-19} \mathrm{GeV}$ & $\pm 0.1 \times 10^{-19} \mathrm{GeV}$ \\
\hline$\pi^{+} \pi^{-}$ & $\pi^{+} \pi^{-}$ & $\gamma$ & $\begin{array}{c}(1.1 \pm 2.5) \times 10^{-21} \mathrm{GeV} \\
\quad(\text { compl. pos. hyp. }) \\
(0.7 \pm 1.2) \times 10^{-21} \mathrm{GeV}\end{array}$ & $\begin{array}{l} \pm 0.2 \times 10^{-21} \mathrm{GeV} \\
\pm 0.1 \times 10^{-21} \mathrm{GeV}\end{array}$ \\
\hline$\pi^{+} \pi^{-}$ & $\pi^{+} \pi^{-}$ & $\Re \omega$ & $\left(-1.6_{-2.1}^{+3.0} \pm 0.4\right) \times 10^{-4}$ & $\pm 2 \times 10^{-5}$ \\
\hline$\pi^{+} \pi^{-}$ & $\pi^{+} \pi^{-}$ & $\mathfrak{I} \omega$ & $\left(-1.7_{-3.0}^{+3.3} \pm 1.2\right) \times 10^{-4}$ & $\pm 2 \times 10^{-5}$ \\
\hline$K_{S, L} \rightarrow \pi e v$ & & $\Delta a_{0}$ & (prelim.: $\left.(0.4 \pm 1.8) \times 10^{-17} \mathrm{GeV}\right)$ & $\pm 2 \times 10^{-18} \mathrm{GeV}$ \\
\hline$\pi^{+} \pi^{-}$ & $\pi^{+} \pi^{-}$ & $\Delta a_{Z}$ & (prelim.: $\left.(2.4 \pm 9.7) \times 10^{-18} \mathrm{GeV}\right)$ & $\pm 7 \times 10^{-19} \mathrm{GeV}$ \\
\hline$\pi^{+} \pi^{-}$ & $\pi^{+} \pi^{-}$ & $\Delta a_{X}, \Delta a_{Y}$ & $\left(\right.$ prelim.: $\left.<9.2 \times 10^{-22} \mathrm{GeV}\right)$ & $\pm 4 \times 10^{-19} \mathrm{GeV}$ \\
\hline
\end{tabular}

\section{Conclusions}

The neutral kaon system constitutes an excellent laboratory for the study of the $C P T$ symmetry and the basic principles of quantum mechanics. Several parameters related to possible $C P T$ violations, including decoherence and Lorentz symmetry breaking effects, have been measured, in some cases with a precision reaching the interesting Planck scale region. Simple quantum coherence tests have been also performed. All results are consistent with no violation of the $C P T$ symmetry and/or quantum mechanics.

A $\phi$-factory represents a unique opportunity to push forward these studies. It is also an ideal place to investigate the entanglement and correlation properties of the produced $\mathrm{K}^{0} \overline{\mathrm{K}}^{0}$ pairs. The KLOE physics program is going to be continued (KLOE-2), and improvements are expected in almost all present limits.

\section{Acknowledgments}

I would like to thank all the organizing committee, and in particular Takashi Kaneko and 
Takeshi Komatsubara for the invitation to this very interesting and well organized conference, and the pleasant stay in Tsukuba.

\section{References}

[1] G. Lueders, Ann. Phys. (NY) 2 (1957) 1, reprinted in Ann. Phys. (NY) 281 (2000) 1004.

[2] W. Pauli, Exclusion principle, Lorentz group and reflexion of space-time and charge in Niels Bohr and the development of physics, edited by W. Pauli, Pergamon, London, 1955, p.30.

[3] J. S. Bell, Proc. R. Soc. London A 231 (1955) 479

[4] R. Jost, Helv. Phys. Acta 30 (1957) 409.

[5] O. W. Greenberg, Phys. Rev. Lett. 89 (2002) 231602.

[6] O. W. Greenberg, Found. Phys. 36 (2006) 1535 (arXiv: hep-ph/0309309).

[7] S. Hollands, Commun. Math. Phys. 244 (2004) 209.

[8] C. Amsler et al., Particle Data Group, Phys. Lett. B667 (2008) 1.

[9] Handbook on neutral kaon interferometry at a $\phi$-factory, A. Di Domenico ed., Frascati Physics Series 43, INFN-LNF, Frascati, 2007.

[10] A. Angelopoulos et al., CPLEAR collaboration, Eur. Phys. J. C 22 (2001) 55.

[11] J.S. Bell and J. Steinberger, Proc. Oxford Int. Conf. on Elementary Particles (1965).

[12] F. Ambrosino et al., KLOE collaboration, JHEP 12011 (2006).

[13] see E. Blucher, these proceedings

[14] M. Palutan, talk given at the Flavianet Kaon Workshop, 12-14 June 2008, Capri, Italy.

[15] M. Adinolfi et al., KLOE collaboration, Nucl. Instr. and Meth. A 488 (2002) 51.

[16] M. Adinolfi et al., KLOE collaboration, Nucl. Instr. and Meth. A 482 (2002) 364.

[17] F. Ambrosino et al., KLOE collaboration, Phys. Lett. B 642 (2006) 315.

[18] A. Di Domenico and the KLOE collaboration, Journal of Physics: Conf. Series 171 (2009) 012008.

[19] R. A. Bertlmann, W. Grimus, B. C. Hiesmayr, Phys. Rev. D 60 (1999) 114032.

[20] A. Apostolakis et al., CPLEAR collaboration, Phys. Lett. B 422 (1998) 339.

[21] A. Go et al, Belle collaboration, Phys. Rev. Lett. 99 (2007) 131802.

[22] S. Hawking, Commun. Math. Phys. 87 (1982) 395.

[23] R. Wald, Phys. Rev. D 21 (1980) 2742.

[24] J. Ellis, J. S. Hagelin, D. V. Nanopoulos, M. Srednicki , Nucl. Phys. B 241 (1984) 381.

[25] J. Ellis, J. L. Lopez, N. .E. Mavromatos, D. V. Nanopoulos, Phys. Rev. D 53 (1996) 3846.

[26] R. Adler et al., CPLEAR collaboration, Phys. Lett. B 364 (1995) 239.

[27] F. Benatti, R. Floreanini, Nucl. Phys. B 488 (1997) 335; Nucl. Phys. B 511 (1998) 550; Phys. Lett. B 468 (1999) 287.

[28] J. Bernabeu, N. Mavromatos, J. Papavassiliou, Phys. Rev. Lett. 92 (2004) 131601. 
[29] J. Bernabeu, N. Mavromatos, J. Papavassiliou, A. Waldron-Lauda, Nucl. Phys. B 744 (2006) 180.

[30] V. A. Kostelecký, Phys. Rev. Lett. 80, 1818 (1998).

[31] V. A. Kostelecký, Phys. Rev. D 61, 016002 (1999).

[32] V. A. Kostelecký, Phys. Rev. D 64, 076001 (2001).

[33] A. Di Domenico, (KLOE collaboration), CPT and Lorentz Symmetry IV, V.A. Kostelecký, ed., World Scientific, Singapore, 2008

[34] M. Testa et al., KLOE collaboration, Proceedings of the XLIII Rencontres de Moriond, Electroweak Interactions and Unified Theories, 1-8 March 2008, La Thuille, Italy

[35] H. Nguyen, (KTeV collaboration), in CPT and Lorentz Symmetry II, V.A. Kostelecký, ed., World Scientific, Singapore, 2002.

[36] B. Aubert, et al., BABAR collaboration, Phys. Rev. Lett. 100 (2008) 131802.

[37] R. Beck et al, KLOE-2 collaboration, Expression of interest for the continuation of the KLOE physics program at DAФNE upgraded in luminosity and in energy, March 31, 2006, available at http://www.lnf.infn.it/lnfadmin/direzione/ roadmap/LoIKLOE.pdf.

[38] R. Beck et al, KLOE-2 collaboration, A proposal for the roll-in of the KLOE-2 detector, LNF-07/19(IR), INFN-LNF, Frascati, 2007.

[39] P. Branchini, these proceedings. 